DESIGN OF EXPERIMENTS FOR LIGHT SPEED INVARIANCE TO MOVING OBSERVERS

\title{
Design of experiments for light speed invariance to moving observers
}

Revised 2/22/2021 08:08:00

\author{
Qian Chen \\ International Education Foundation \\ 4667 Highland Ct, Grandville, MI, USA 49418 \\ e-mail: georgechen@iefstudy.org
}

\begin{abstract}
The principle of the constancy of the velocity of light, which stated that the light velocity is invariant to the motion of the emitter, was well established and directly proven by experiments. Interestingly, the further assumption that the light velocity is independent of the motion of the observer was, arguably, never directly proven by any experiment for a century. This paper proposed the design of two experiments to directly test this assumption, which tried to address some perceived technical difficulty in such experiments. One is to directly measure the light speed as to moving sensors. The experiment setup is designed in such a way that the concerns of time synchronization and dilation can be avoided. Another experiment is to test the isotropy of the light speed to a moving particle in the electromagnetic accelerator by measuring the momentum to acceleration ratio. The experiment result, if positive, will provide direct and solid proof of the assumption. Otherwise, it may imply a need for further investigation. Since the light speed invariance to moving observers is a key assumption of some fundamental physical theory, either way, the experiments will have significant meanings.
\end{abstract}

Keywords: Light velocity, Special Relativity, Time dilation, Time synchronization, Sagnac effect, MassEnergy relationship, Particle acceleration, Michelson-Morley experiment, Asymmetry Theory

\section{INTRODUCTION}

The second postulate of Special Relativity (Einstein 1905 [1, 2, 3]), "the principle of the constancy of the velocity of light", stated: "in empty space, light is always propagated with a definite velocity $\mathrm{V}$ which is independent of the state of motion of the emitting body". This principle was experimentally validated by, the uniformity of the timing signature of the binary $\mathrm{x}$-ray pulsar system Her X1 [8], the measurement of the speed of gamma rays emitted by fast pions [11], and the laboratory experiments with moving optical elements [12]. However, the further implicit inference in Special Relativity that the light velocity is also independent of the motion of the observer due to the "principle of relativity" [1], was, arguably, never directly proven by any experiment for more than 100 years. There are some experiments claimed to be proof of the assumption, but all are based on inference, not direct proof. Michelson-Morley-experiment [7] was claimed as proof that the light speed is invariant to the directions of moving observers. In fact, the null-result of the M-M experiment only proved that the light speed variance, if any, is much smaller than the earth's speed, $30 \mathrm{~km} / \mathrm{s}$, which is the theoretic prediction of the ether theory. Other important theories of light velocity include the ether theory (Lorentz 1904 [6]) which is empirically equivalent to Special Relativity, and the emission theory (Ritz 1908 [9]) which contradicted the principle of constant light speed [10].

Because the invariance of light speed is the key assumption for some fundamental physical theory, the search for direct experimental proof is of significant importance. Although the reason that no one ever tried to implement a direct experiment is not fully clear, the technical difficulty of clock synchronization and time dilation is perceived as part of the reason.

Two experiments are designed to directly test the invariance of light speed as to moving observers. The first is originated from the Sagnac effect $[15,16]$. The Sagnac effect questioned the assumption of the invariance of light speed to the moving observers. Special Relativity can not explain the Sagnac effect and the contradiction was attributed to the rotating/accelerating frame of the experiment, and an explanation was provided with General relativity [17]. Hence, the goal of the experiment design is to bring the Sagnac effect into an inertia frame, which is in the scope of Special Relativity. The experiment is designed to directly measure the travel times of a light beam to two detectors moving at the same constant speed, i.e. no acceleration, in opposite directions. The experiment is also set up so that the concerns of time synchronization and time dilation can be minimized. A high-speed camera can be deployed to capture the exact moments of photon detection by both sensors and determine if there is a sequence of events. Furthermore, a reference setup is leveraged to confirm the time synchronization of clocks.

The second experiment is to test the isotropy of the light speed as to a moving particle in the electromagnetic accelerator. Let's measure the ratio of the momentum applied by the accelerator to the acceleration of the particle, which is expected to decrease during the acceleration. First, the particle is accelerated to a high speed, say $0.5 c$. Then 


\section{DESIGN OF EXPERIMENTS FOR LIGHT SPEED INVARIANCE TO MOVING OBSERVERS}

the direction of the accelerator is reversed to decelerate the particle, i.e. the direction of the particle's velocity is reversed. If the speed of the electromagnetic wave is isotropic to the moving particle, the momentum to acceleration ratio shouldn't have any immediate impact on the ratio, because the relativistic mass in Special Relativity is the same for positive and negative velocity.

\section{THEORY BACKGROUND}

\section{A. The Sagnac Effect}

The Sagnac effect [13] shows that two light beams, sent clockwise and counterclockwise around a closed path on a rotating disk, take different time intervals to travel the path. It can be extended to a FOG [14] with

$$
\Delta t=2 v L / c^{2}
$$

where $v$ is the detector's speed, $L$ is the distance. The Sagnac Effect contradicted the assumption that the light velocity is independent of the motion of the observer.

Special Relativity attributed the contradiction to the rotating/accelerating frame of the Sagnac effect experiment, i.e. outside of the scope of Special Relativity, and an explanation was provided with General Relativity[11].

The idea is to bring the Sagnac effect experiment into the set-up of an inertia framework, i.e. take out the rotation. A transient light beam is sent to two detectors moving at the same constant speed, i.e. no acceleration, in opposite directions. The travel times of the light beam to the detectors are measured. If the travel time difference is 0 , the result will directly prove the invariance of light speed as to moving observers. If under this inertia system setup, the measured time difference is still $\Delta t=2 v L / c^{2}$ as predicted in [15], a further investigation of the assumption may be required.

\section{B. Time synchronization and dilation}

The synchronization of Time in [1] stated, "Let a ray of light start at the "A time" $t_{A}$ from A towards B, let it at the "B time" $t_{B}$ be reflected at $\mathrm{B}$ in the direction of $\mathrm{A}$, and arrive again at $\mathrm{A}$ at the "A time" $t_{A}^{\prime}$, the two clocks synchronize if

$$
t_{B}-t_{A}=t_{A}^{\prime}-t_{B}
$$

For an observer moving with speed $v$, we must satisfy

$$
t_{B}-t_{A}=\frac{r_{A B}}{c-v} \text { and } t_{A}^{\prime}-t_{B}=\frac{r_{A B}}{c+v}
$$

The contradiction between these equations is resolved with the "time dilation" in Special Relativity [1]

$$
\Delta t^{\prime}=\Delta t / \sqrt{1-v^{2} / c^{2}}
$$

Hence, it is important to consider the time dilation and synchronization concerns in the experiment. In the experiment setup, the light source and the detectors are placed in the same initial place. Both detectors are moving at the same speed so that if there is a time dilation effect, it will be the same.

\section{The Momentum to Acceleration ratio}

It is well known that a particle in the accelerator becomes difficult to accelerate when its speed increases and it is impossible to approach the light speed $c$. That means that the ratio of the momentum applied by the accelerator to the acceleration of the particle decreases when the speed increases. The explanation of Special Relativity [10] is the relativistic mass increase for a moving particle:

$$
m_{\text {rel }}=m_{0} / \sqrt{1-v^{2} / c^{2}}
$$

This follows the equation for the ratio of momentum to acceleration:

$$
\frac{d P}{P} / \frac{d v_{o}}{v_{o}}=1 / \sqrt{1-v^{2} / c^{2}}
$$

(5) shows that the ratio increases when the speed increases and approaches infinity when $v$ approaches $c$. It also shows that the ratio is the same for a particle with the same speed in opposite directions, i.e. acceleration vs. deceleration. For example, the ratio is the same for $v=0.5 c$ and $v=-0.5 c$. This prediction will be tested in the experiment.

\section{EXPERIMENTS DESIGN}

Two experiments are designed to directly test if the light speed is independent of the motion of observers.

\section{A. Light velocity to moving observers}

\section{EXPERIMENT SETUP}

A transient light source and two moving light sensors are in the same initial position and connected to the same precise digital scope with cables of equal length, see FIG. 1. A high-speed camera is an enhancement to capture the exact moments when the photon hits the sensors. This setup is to avoid the time synchronization concern. Both sensors are moving at a constant speed $v$ but in opposite directions. Hence if there is a time dilation effect, it should be the same for both detectors. The light can be reflected multiple times to increase the path length and improve accuracy.

To further minimize the concern of the time/clock synchronization, a similar reference setup is leveraged to calibrate the time measurement, see FIG. 2. In this reference we use two light sources moving at the same speed $v$ but in opposite direction. The detection times of both light beams are recorded. Since the light-speed invariance to the motion of the light source is well-proven, 


\section{DESIGN OF EXPERIMENTS FOR LIGHT SPEED INVARIANCE TO MOVING OBSERVERS}

any measured detection time difference can be attributed to the time or clock drift due to the movement at speed $v$. Hence the results from this reference setup can be used to calibrate any potential time or clock drift in the experiment.

EXPERIMENT STEPS

It is controlled that light is emitted when both sensors coincide in the same initial position. The emitted light will be reflected by a mirror back to the moving sensors. The detection times of both sensors are measured by the digital scope. Besides, the high-speed camera will capture the exact moments when the photon hits the sensors.

The experiment should always be repeated with the sensors switching directions. For example, the first time is sensor $1 v$ and sensor $2-v$, the next time, it is sensor $1-v$ and sensor $2 v$. This helps distinguish the time difference due to the clock drift from that due to the motion. The experiment should be repeated many times and with different velocities $v$. Data analysis, for example, statistics, should be applied to reduce random errors.

RESULT ANALYSIS

If the light speed is independent of the motion of moving observers, both sensors shall detect the light at the same moment. Therefore, if the experiment results show there is no time difference detected by the digital scope and the camera, the experiment proves the light-speed invariance to moving observers.

However, if a time difference $\Delta t=2 v L /\left(c^{2}-v^{2}\right)$ is detected, i.e. same as the Sagnac effect as predicted in [15], a further investigation is warranted.

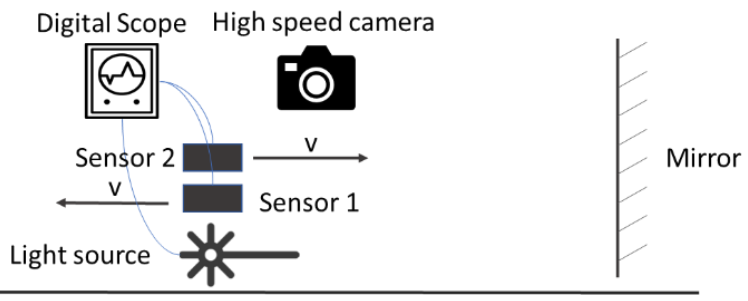

FIG. 1. Setup for the light velocity to moving observers

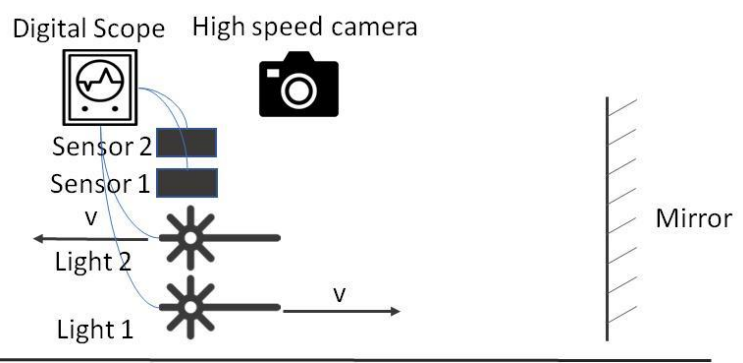

FIG. 2. Reference setup for time calibration

\section{B. Momentum to acceleration ratio Experiment}

\section{EXPERIMENT SETUP}

This experiment is to accelerate a particle, for example, an electron, in a linear electromagnetic accelerator, which could instantly switch the direction of acceleration. The devices are set up so that the momentum applied by the accelerator and the particle's speed can be measured and recorded. The measured data is then used to calculate the momentum to acceleration ratio in a time plot.

\section{EXPERIMENT STEPS}

First, the particle is accelerated to a high speed, say $0.5 \mathrm{c}$ . Then the direction of the accelerator is reversed to decelerate the particle. The moment applied by the accelerator and the particle's speed is measured continuously during the process. The experiment can be repeated many times and with different types of particles and different particle speeds. Data analysis, for example, statistics, can be applied to reduce random errors. The result is the time plot of the momentum to acceleration ratio.

\section{RESULT ANALYSIS}

As predicted by (5) of Special Relativity, the momentum to acceleration ratio should keep constant at the exact moment of the reversal of acceleration direction. If the time plot shows that the momentum to acceleration ratio is continuous at the time of direction reversal, it proves that the electromagnetic wave accelerating the particle is isotropic to the motion of the particle. If the time plot shows there is a significant increase, jump, of the ratio at the time of direction reversal, as predicted in [15], a further investigation is warranted.

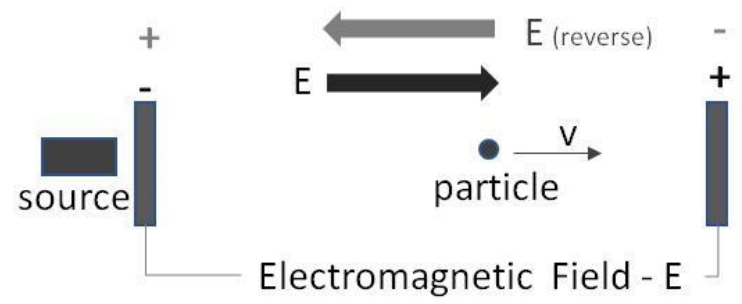

FIG. 3. Setup for the particle acceleration/deceleration

\section{CONCLUSIONS}

Two experiments were designed to directly test the invariance of light speed to moving observers, which is fundamental to many theories. The results should be able to confirm the assumption, hence closing a long-time loophole. Or a further investigation is warranted. Either way, these experiments are of significant importance.

It is best if these experiments can be carried out by independent groups so that the results are more convincing.

\section{ACKNOWLEDGEMENTS}

This research is sponsored by the International Education Foundation. 
[1] A. Einstein, On the Electrodynamics of Moving Bodies, Annalen der Physik, 17, 891-921 (1905).

[2] A. Einstein, Does the Inertia of a Body Depend Upon its Energy-Content?, Annalen der Physik, 323 (13): 639641 (1905).

[3] A. Einstein, On the Possibility of a New Test of the Relativity Principle, Annalen der Physik, 328 (6): 197198 (1907).

[4] A. Einstein, Generalized theory of relativity, the anthology 'The Principle of Relativity', 94, University of Calcutta (1920).

[5] Langevin, Paul, Sur la théorie de la relativité et l'expérience de M. Sagnac, Comptes Rendus. 173: 831-834 (1921).

[6] H. Lorentz, Electromagnetic phenomena in a system moving with any velocity smaller than that of light, Proc. R. Neth. Acad. Arts Sci. 6: 809-831 (1904).

[7] A. Michelson and E. Morley, On the Relative Motion of the Earth and the Luminiferous Ether, Am. J. Sci. 34 (203): 333-345 (1887).

[8] K. Brecher, Phys. Rev. Lett., 39, 1051 (1977).

[9] W. Ritz, Recherches Critiques sur les Theories Electrodynamiques de Cl. Maxwell et de H.-A. Lorentz, Archives des Sciences physiques et naturelles 36: 209 (1908).

[10] J. G. Fox, Evidence Against Emission Theories, Am. J. Phys. 33 (1): 1-17 (1965).

[11] F. Alvaeger et al., Phys. Lett. 12, 260 (1964).

[12] G. C. Babcock et al., J. O. S. A. 54, 147 (1964).

[13] G. Sagnac, C. R. Acad. Sci. Paris 157, 708 (1913).

[14] R. Wang et al., Physics Letters A. 312 (1-2): 7-10 (2006).

[15] Q. Chen, Asymmetry Theory derived from the principle of constant light speed, submitted to Nature Phys., doi:10.31219/osf.io/6a74s (2021). 